\title{
D’une actualité dans le roman maghrébin
}

\author{
Abdelaziz AMRAOUI \\ Université Cadi-Ayyad Marrakech (Maroc) \\ a.amraoui@uca.ac.ma
}

Recibido: 04/07/2016

Aceptado: 27/09/2016

\section{Résumé}

Dans ces temps de mélange, la trinité - l'individu, la communauté, la société - est de plus en plus mobile, en allant à la rencontre d'autres rassemblements et en accueillant d'autres. Cette fusion, dans l'ère du soupçon et l'ère de doute va bouleverser la donne. En conséquence, des îlots de résistance apparaîtront formant un archipel de conscience tentaculaire détruisant les normes et les doxas sur lesquelles repose la société. L'on passe de l'individu à une communauté fermée pour basculer une société entière dans le chaos...

La littérature s'implique dans l'affaire et raconte le phénomène.

À partir de deux romans, respectivement de l'Algérien Samir Bachi et le Marocain Mahi Binebine, Tuezles tous ${ }^{1}$ et Les Étoiles de Sidi Moumen ${ }^{2}$, nous essayerons d'étudier le processus de construction d'intégrisme quand l'intégration au sein de la société fait défaut, sans par ailleurs que cela soit l'apanage d'une catégorie au détriment d'une autre. Le communautarisme cède le pas au sectarisme à l'intérieur d'une même société, somme toute, unie...Ainsi, sera-t-il question de voir comment un archipel terroriste se crée quand la convivialité ne peut plus mettre en œuvre une « utopie concrète » d'une société, créée à l'image d'un Dieu Clément...

Mots clés : terreur, littérature maghrébine, Casablanca, New York, religion.

\section{La actualidad de la novela magrebí}

\section{Resumen}

En estos tiempos de mezcla, la trinidad -individuo, comunidad, sociedad- es cada vez más móvil y va al encuentro de otras formaciones, y acoge a otras. Esta fusión, en estas zonas de sospecha, y época de duda, supone cambios fundamentales. En consecuencia, las zonas de resistencia aparecerán formando un archipiélago de conciencia tentacular susceptible de destrozar las normas y la doxa que sustenta la sociedad. El individuo es remplazado por una comunidad cerrada para empujar a toda una sociedad hacia el caos...

La literatura forma parte de este proceso y se ocupa también de relatarlo.

A partir de las novelas del argelino Samir Bachi y del marroquí Mahi Binebine, Tuez-les Tous y Les Étoiles de Sidi Moumen respectivamente, vamos a tratar de estudiar el proceso de construcción del integrismo cuando fracasa la integración dentro del sociedad, sin que por lo demás, ello sea la prerrogativa de una clase sobre otra. El comunitarismo da paso al sectarismo dentro de una misma sociedad. Así, se tratará de ver cómo se constituye un archipiélago terrorista cuando la convivialidad ya

\footnotetext{
${ }^{1}$ Paru chez Gallimard, Paris, 2006.

2 Paru chez Flammarion, Paris, 2010 et chez Le Fennec, Casablanca, 2010.
} 
no es capaz de poner en práctica una "utopía concreta" de una sociedad creada a la imagen de un Dios Clemente.

Palabras clave: terror, literatura magrebí, Casablanca, Nueva York, religión.

\title{
Topicality in the Maghrebi Novel
}

\begin{abstract}
The current threefold fusion of individual, community, and society is increasingly mobile and fluid, especially in its encounter with the other. In these times of suspicion and doubt, this fusion will help to upend our given assumptions. Islands of resistance appear, forming archipelagos of consciousness that undermine the standards and postulates by which society lives. Literature is involved in this process. Using two novels as material for analysis-Tuez-les Tous, by the Algerian Samir Bachi, and Les Étoiles de Sidi Moumen, by the Moroccan Mahi Binebine - this essay attempts to study the process of fundamentalism, as it builds up when social integration fails. Communitarianism gives way to sectarianism within the same society, in what is, after all, an altered form of unity. The question that then remains is how a terrorist archipelago emerges when conviviality cannot implement a God-inspired, concrete utopia in a given society.
\end{abstract}

Keywords: novel, Casablanca, New York, religion.

Sommaire : De Tuez-les tous. Des Etoiles de Sidi Moumen. Des deux auteurs. De la narration. De la violence... ... à la terreur. Du divin. Du rapport avec l'État. Médias et Histoire. En guise de conclusion.

\section{Referencia normalizada}

Amraoui, A. (2016). «D'une actualité dans le roman maghrébin ». Thélème. Revista Complutense de Estudios Franceses, Vol. 31, Núm. 2 : 207-225.

http://dx.doi.org/10.5209/rev_THEL.2016.v31.n2.53138

« Celui qui a tué un homme qui lui-même n'a pas tué ou qui n'a pas commis de violence sur la terre est considéré comme ayant tué tous les hommes. » Coran, sourate V.

Le 11 septembre 2001 est une date charnière, non seulement par rapport à des considérations géopolitiques et géostratégiques, en relation avec le terrorisme international, mais aussi en relation avec son impact sur le monde artistique et littéraire. Le Maghreb n'est pas en reste. Salim Bachi, l'Algérien, et Mahi Binebine, le Marocain, vont ouvrir l'espace de tous les dangers, sans sécurité de notre postmodernité très agitée, post-modernité qui replonge le monde dans une deuxième « ère de soupçon » avec son lot d'interrogations et de doutes. Ils vont percer, et ce ontologiquement, ce mystère de la construction de l'orthodoxie aveugle et aveuglante, chez des personnages totalement décalés du monde dans lequel ils vivent, 
et dont l'éducation et les origines sont foncièrement différentes. Au nom d'une lecture de l'Islam, au diapason de la réalité, des vengeurs de la cause musulmane vont semer la terreur, d'un côté aux États Unis, puis, dans une terre de l'Islam, le Maroc, contre des musulmans et des non-musulmans !

\section{De Tuez-les tous (désormais TLT)}

Récit hallucinant par sa charge référentielle et sa densité, ce qui explique la brièveté du texte, et le rythme tourbillonnant dicté par la folie meurtrière du personnage qui laisse apparaître une violence destructrice et un ressassement inlassable, mais aussi (et surtout) par ce personnage héros dont la puissance fut nourrie d'incroyance et de détermination, forgée par une organisation d'incrédules à laquelle il a adhéré suite aux multiples rejets et échecs, notamment celui commis par son ex-épouse et son état d'orphelin :

Orphelin, il avait erré à Paris, la ville lumières éteintes. Puis, il avait été adopté par l'Organisation. Il suivit leur enseignement dans la mosquée où on le laissait dormir, lui qui n'avait plus ni feu ni lieu. Ils l'avaient nourri, protégé et il avait grandi, devenant immense par son incroyance et sa détermination (TLT, $2006: 26$ ).

Tous ces déboires entraîneront le chaos dans sa vie et l'emmèneront au fin fond des ténèbres entre les décombres des tours jumelles à New York.

\section{Des Etoiles de Sidi Moumen (désormais LSDM)}

En l'espace de quelques minutes, cinq attentats-suicides quasi simultanés vont frapper Casablanca le 16 mai 2003. Chaque fois un lieu symbolique. La cible des kamikazes était des hôtels et des restaurants fréquentés par les étrangers. 45 morts dont trois français et deux espagnols, une soixantaine de blessés. Ceux qui se cachent derrière ces exactions sont des jeunes recrutés dans le lit de tous les possibles terroristes : Sidi Moumen, le grand bidonville de la métropole, abject lieu de survie, d'où sont venus de jeunes gens qui mirent fin en cette nuit noire au mythe du Maroc immunisé contre le terrorisme.

\section{Des deux auteurs}

Tous deux sont des témoins de leur temps, et ne sont pas seulement dans le pur littéraire. La littérature, pour eux, est aussi cette non-distance avec le réel qui va être mis en spectacle, et de quelle manière ! L'écriture va mettre le roman en porte à faux avec la fiction.

Binebine et Bachi vont voir dans l'endoctrinement - le dernier en vogue - par la religion, selon telle ou telle secte ou exégèse, l'origine du mal de l'individu et de la société. Le mal allait être créé par celle-là même qui devait semer la paix. La religion, ou du moins ce qu'on en a compris, est cette entrée en matière du mal dans le monde. 
De son statut résiduel et insulaire, la violence religieuse va devenir continentale et va propager sa haine sur et envers l'Autre.

\section{De la narration}

La narration raconte le cheminement et le processus de déchaînement de la violence tue et absorbée d'un ensemble de personnages, avec, en fil d'Ariane, la manière et l'art avec lesquels les individus deviennent des machines à tuer, des Kamikazes.

D'une part, un pilote d'avions avec un niveau d'instruction élevé, dans le texte de Bachi ; et de jeunes banlieusards de Casablanca, sans instructions, sans passé, sans avenir, mais, foncièrement porteurs de leur mort et celle de bien d'autres. Ici, un narrateur omniscient, tantôt hétérodiégétique, avec un sens aigu de la critique, tantôt homodiégétique. Là, le narrateur est auto-homodiégétique racontant, d'outre-tombe, dans l'attente de son Jugement et de son admission au Paradis, son parcours de kamikaze, à la première personne « je »: «Et j'attends toujours les anges » (LESM : 150). Yachine, c'est le nom du narrateur, va mettre devant le lecteur son vécu passé sous forme d'une conscience posthume amère en train de présentifier des événements passés comme s'ils nous étaient contemporains. Avec ce roman, Mahi Binebine va réussir un coup de génie quand il mettra la narration entre les mains d'un mort pour « revivre, par une sorte d'[introspection rétrospective], un choix que nous avons déjà fait» (Foulquié, 1972: p. 31) dans un projet tragique qui rendra compte d'événements marquants. Les moments choisis seront constitutifs d'un processus cognitif et psychologique loin d'être l'aboutissement d'une mûre réflexion.

\section{De la violence...}

La violence est paradoxalement entreprise dans le sens d'une arme défensive contre cet Autre diabolisé et élevé au rang d'hérétique, et par extension, toute son engeance. Qui ne me ressemble pas est passible d'être mon ennemi, et à plus forte proportion, celui de l'autre religion. Cette réalité est d'autant plus actuelle aujourd'hui qu'elle ne l'était dans le passé.

La violence est un exutoire. On y est parce qu'on a tout perdu ou, cas extrême, rien eu depuis le début, comme c'est le cas de Khaled de Tuez-les tous. L'individu atteint ... de violence emprunte un désert sans détour. Une suite d'échecs et de rencontres hasardeuses va plonger l'individu dans le gouffre de non-retour, mais aussi de la reconnaissance, ce qui paraît contradictoire. Ceci explique la manière avec laquelle le kamikaze agit. En augmentant l'horreur des exactions, il veut se différencier de toute autre personne commettant un délit ou un meurtre. Ceci paraît mineur, et la juridiction y afférente est aussi plus grande que celle allouée à ce type de délit. L'atrocité de l'acte est un gage de réussite posthume, dont le monde va parler. Et tant qu'on en parle, la campagne publicitaire, bon escient mal escient, est entamée. Et il la réussira. 
L'un abattra un avion « le Boeing 767 de la compagnie American Airlines sur les deux tours les plus orgueilleuses de l'humanité. Les yeux grands ouverts » (TLT : 27) et les autres vont semer la terreur à Casablanca. Pas d'attaches, pas d'avenir. Le peu de passé qu'ils ont leur renvoie une image éclatée de soi sans profondeur; rien que des bribes démultipliées dégageant un malaise existentiel, quasi inhumain. La souffrance suffoquée et suffocante, à la fois, va ronger les personnages-bombes jusqu'à épuisement de la vie, qui va être perçue de l'intérieur comme un spectacle sui-generis donnant sur soi, pour qu'enfin de compte, après l'exécution de l'acte ultime, elle soit exposée au monde entier dans une scène inouïe de désolation inimaginable à dimension planétaire. Ce destin chaotique échappe à tout entendement, il est cette impression, cette révélation qui va les dessécher et les abstraire de leur monde qui se résumera, désormais, en un « cœur qui s'écœure » (Verlaine, 1991 : 127).

La violence de l'opération doit rester dans la mémoire collective. Il faut qu'elle perdure dans les annales comme sont restées toutes les violences à l'égard des peuples impies relatés dans les Livres. L'horreur et l'ampleur sont telles que l'acte en luimême doit susciter à la fois l'indignation mais aussi, paradoxalement, la surprise. C'est la main de Dieu qui s'abat sur les infidèles et ce n'est que Justice! Pas de prémisses, il faut agir, frapper alors que le dragon dort dans cette sérénité qui est la sienne, lui qui n'a peur de rien :

Que des cités nous avons détruites ! Notre rigueur s'est abattue sur elles durant le sommeil de la nuit ou le repos de la journée et lorsque notre rigueur s'est abattue sur elles, leur seul cri d'appel a été : « Oui ! Nous avons été injustes ! »3 (LTL, $2006: 131)$.

Les médias, comme des enragés et touchés dans leur dignité, puisqu'ils représentent un système politique, vont exploiter l'événement pour engager un discours alarmiste mettant en alerte et en haleine tout le monde contre une poignée d'individus - et indirectement - ce qu'ils représentent : l'islam ou l'islamisme. L'attentat, à l'image de la tendance médiatique, va être diffusé quasi-instantanément. La réalité s'invite à la téléréalité. Le spectacle est au rendez-vous, le spectacle est événementiel.

La violence est un thème universel, elle n'est l'apanage ni d'un temps ni d'un espace, partout elle est et partout elle restera. Elle demeure singulière et se nourrit de représentations mentales qui touchent la zone la plus enfouie de l'individu. Elle est née avec l'Homme, comme si le monde n'était jamais, et ce depuis la Genèse, idéal. Elle est, apparemment, un mal nécessaire difficile à être évincé de cette nature belliqueuse aussi vieille que l'Homme lui-même.

\footnotetext{
${ }^{3}$ Les Livres parlent du peuple de Loth, Sodome et Gomrrhe qui ont été anéantis par la volonté de Dieu, mais aussi suite aux fléaux qui se sont abattus sur l'Égypte, le déluge, les sauterelles, la vermine, les grenouilles et le sang.
} 
Sa représentation, néanmoins, diffère d'un écrivain à un autre mais aussi d'un temps à un autre. Comme la littérature est le souffle de la vie, elle n'a jamais été que de la réalité et du vécu de l'Homme. Elle va rendre compte, à sa façon bien sûr, du monde et de ses changements. La violence en est une partie; que ce soit la guerre ou autres manifestations de la violence plus locale: violence conjugale, contre les enfants, elle est une dominante thématique qui ne quittera jamais la fiction. L'Histoire de l'Homme, dans sa version monothéique, est d'abord l'expression d'une enfreinte à une interdiction, considérée comme violence contre le libre arbitre qui le distingue des autres créatures. Le deuxième épisode est ce crime originel perpétré par un fils d'un envoyé de Dieu à l'encontre de son propre frère. Les trois Livres de la révélation en font le récit.

Son caractère permanent et presque hiératique fait de la violence un thème transfrontalier et transgénérique. Les premiers textes en français manifestent cet intérêt pulsionnel à parler de la violence et à parler des conflits. La Chanson de Roland en est l'illustre exemple.

La violence vue par ses détracteurs est une catharsis vindicative qui prend ses sources dans des fondements pseudo-religieux (œil pour œil...) à échelle planétaire. Sur ce, la violence est « une structure constante du phénomène humain » (Maffesoli, 2001 : 17). C'est un consta(n)t anthropologique reposant quasiment toujours sur

une menace sur le territoire réel ou symbolique que l'on contrôle (un espace de terre, mais aussi un objet possédé, une identité qu'on pense menacée, ou une personne sur laquelle on estime avoir des droits) et dont on craint d'être dépossédé (Onfray, $2001: 187)$.

\section{... à la terreur}

Que ce soit au niveau individuel ou collectif dans le cadre de "l'Organisation", l'orthodoxe « conforme au dogme, à la doctrine d'une religion » (Le Petit Robert de la langue française 2012, Edition électronique) va vivre isolé, retiré, retranché...L'espace qu'il se confectionne est à l'image de ses idées et idéaux. C'est un archipel dont la frontière est un énorme désert au diapason de la vie courante.

La conquête pour la territorialisation de l'espace urbain implique des constructions idéologiques et se transforme alors en épreuve de force où chacun tente de délimiter des frontières plus ou moins réelles, combinées à des représentations collectives. Au-delà des simples disparités économiques, cet îlot doit être conquis et rien ne vaut mieux qu'un groupe homogène avec un idéal et un objectif communs : « Nous apprîmes à nous serrer les coudes, à refuser tout net l'état de larve auquel nous étions condamnés à perpétuité. Nous savions que les droits ne se donnaient pas, ils s'arrachaient » (LESM : 108).

Dans les deux romans, il y a « une série de lieux surdéterminés pour bien mettre en valeur l'aspect double et circulaire du texte» (Baetens, 2005 : 54). New York d'une part, théâtre de la collision de deux avions sur les tours jumelles, et Sidi Moumen, à Casablanca, qui a été frappée en 2003 par des attentats ayant fait 
beaucoup de morts dans trois sites différents ${ }^{4}$, hauts lieux de la tolérance et de la cohabitation des cultes dans un pays ouvert à toutes les influences. Ce bidonville est l'exemple-type de la paupérisation du Maroc dont la pression démographique des couches urbaines ne va qu'amplifier la situation. Dans ces conditions désastreuses, le bidonville oublié par les pouvoirs publics, et pourtant maintenu dans une léthargie comme sans vie, est constamment en proie au désœuvrement de sa population, victime de toutes sortes d'atteinte à la dignité humaine, mais avide et assoiffée de conditions de vie meilleure et plus respectueuse. Cet état des lieux va favoriser l'expansion d'une idéologie, sous couvert d'un islam populaire attrape-tout, revu et revisité, absorbant les problèmes urgents d'une population à la marge de la société et démunie sur tous les fronts. En tant que ghetto, Sidi Moumen est une fourmilière pour toute espèce de dépravation. Rien n'y sied ; une frontière imaginaire et matérielle cache la cité ${ }^{5}$ à laquelle foncièrement, territorialement et administrativement il appartient. Les habitants du bidonville vivent à la marge et en isolement qu'ils ont dû acquérir une mentalité et un comportement insulaires. En résumé, la pauvreté et la marginalisation zonale ou urbaine ont accéléré les fiefs radicaux à se constituer et à germer, facteurs déterminants pour le recrutement de jeunes.

En fait, le temps et l'espace sociaux sont morcelés par la victoire mondiale d'un marché de la communication et des idées où la diffusion se fait à la vitesse de la lumière. Il s'agit de la naissance d'une nouvelle donne qui veut régner sur les mentalités, lesquelles rencontrent des résistances de la part de personnes s'affichant dans la scène publique et criant haut et fort leur allégeance à leur religion, ou du moins à ce qu'ils en ont compris, après un endoctrinement où les maîtres apprennent à leurs disciples le sens du don, le don de la mort et de l'auto-sacrifice pour une cause fatale. En conséquence, l'attachement à la religion devient plus fort que l'appartenance à une nation; et quand c'est le cas, l'individu ne vivant et n'existant plus de droit, troque sa citoyenneté contre ses convictions religieuses et sa compassion et son humanité contre la haine sans trop se soucier du cadre juridicopolitique dans lequel il vit et sous lequel il doit être, d'autant plus que le sentiment qui le gagne est celui d'être de trop.

L'intéressé a rompu le cordon ombilical qui le lie à cette nation au «Pouvoir voyoucratique » (Khadra, $2014: 125)$ et à ses institutions. Et quand « le pays est aussi fragile qu'un hymen » (Khadra, 2014: 50), il va conquérir ses droits tout en contestant l'idéologie officielle. La désolidarisation de la communauté sera donc perçue comme un désaveu de l'État et une allégeance à une nouvelle autorité qui occultera sa volonté libre, et qui lui recommandera de disposer, à son tour, de la violence légitimée ou rendue légitime, selon tel ou tel prédicateur. L'orthodoxe devient Autre chez lui, devient contingent, différent au sein même de sa société, voire de sa famille comme c'est le cas des personnages du romancier marocain. La

\footnotetext{
${ }^{4}$ Un club géré par un espagnol, un cimetière juif et un hôtel 5 étoiles...

${ }^{5}$ La cité est prise dans le sens d'une agglomération à la périphérie de la ville.
} 
séparation est consommée, elle devient chronique puis létale. La fuite vers l'avant est un gage de soumission à ce nouvel ordre que certains zélotes vont instaurer à l'insu d'un pouvoir démissionnaire et d'une communauté aux abois.

\section{Du divin}

L'ère du divin gagne du terrain. Après la terreur au nom de la République, de la Monarchie ou de l'État de droit tout court, de la guerre, de la démocratie, de la politique... on assiste au retour de la terreur par la religion et les exégèses y afférant. En conséquence, l'aire du divin est sans frontières, elle est à l'image des grandes sociétés dites transfrontalières et sa propagation est fulgurante. Les autres systèmes d'endoctrinement et de ralliement idéologiques peuvent être facilement circonscrits géographiquement pour des raisons relatives aux ordres régissant les États. Pourtant, il y a un seul ordre qui soit réellement et foncièrement fédérateur et séparateur, c'est la religion.

$\mathrm{Au}$ fait, cet endoctrinement est une réaction à la mondialisation-inclusion que subit la société arabe. Et la montée en flèche d'une bourgeoisie, qui ne finit pas de creuser un fossé énorme à l'intérieur de la composante de la société. En contrepartie, des îlots de résistance vont commencer à bâtir les argumentations grâce à quelques prêcheurs et exégètes dissidents qui décideront de créer un autre monde parallèle, un archipel où la haine et l'auto-exclusion sont de mise. La conscience, après coup, d'être différents poussera les disciples à agir aveuglément en espérant satisfaire les Commandements du maitre à penser. Cette opération va rendre compte, en filigrane, de la construction d'un modèle de personne en creux, réplique négative de ce qui existe.

Ils deviennent presque des drones humains téléguidés selon le bon gré d'un zappeur, qui tient dans une main une télécommande et dans l'autre main un chapelet. Ce noyau d'individus, formé à force des acclamations et des hourras au nom d'Allah Akbar, est un groupe dont l'objectif ultime est le « dévoiement de la liberté de pensée, d'opinion ou de religion qui porte atteinte à l'ordre public, aux lois ou aux règlements, aux droits fondamentaux, à la sécurité ou à l'intégrité des personnes » ${ }^{6}$. L'État-nation (Mauss, 1969 : 15), dans ce sens, n'a plus de pouvoir ni sur ce groupe hors-la-loi ni sur son quartier général (Sidi Moumen), et ce n'est que sa référence théologique qui le guide, que le chemin qu'il va emprunter soit compatible ou non avec les lois civiles de son pays. Baudrillard va parler en terme de « déséquilibre» (Baudrillard, 2002 : 16) pour définir cet état de chose qui va jusqu'à devenir l' «anti-dispositif» du système politique et social en vigueur, totalement démissionnaire dans ce territoire, faute du maintien de l'acte contractuel tacite et originel qui devait lier les deux parties,

\footnotetext{
${ }^{6}$ Jean Romain, Rapport de la Commission des pétitions chargée d'étudier la pétition contre les dérives sectaires.
} 
et dont l'assise fondamentale est la justice sociale. Le cosmos social va céder sa place au chaos naissant chez un groupe occulte qui va multiplier les occasions pour mettre à sac « la loi du souverain » qui est, essentiellement, à la recherche de la perfection ${ }^{7}$. Bref, «c'est quand le pouvoir manque que la violence tend à occuper le terrain » (Ricœur, $1991: 26$ ).

Il n'est plus face à cet autre originaire d'un pays que tout sépare du sien; il se trouve plutôt devant un hérétique résidant cette sphère-même que celui-ci a tracé luimême et qui s'appelle Occident. Ce schisme est d'autant plus intéressant qu'il cache cette ancienne haine chrétienne envers l'islam et Mahomet : « La mémoire appartient aux vainqueurs, la mémoire appartient aux Romains » (TLT : 21). Ainsi, plongé dans cette spirale ségrégationniste, le personnage est fidèle à sa lecture du texte religieux, sinon à son apprentissage qui ne s'est fait que dans ce sens. Il est sujet d'une cause qui dépasse l'entendement du citoyen qu'il n'est plus. Sa raison d'être et son moteur sont, désormais, ses convictions religieuses, plus à même de l'aider à habiter le paradis au prix de la multiplication des crimes ici-bas! « Sa nouvelle raison d'être était de nous aider à purifier nos âmes, à nous mettre sur le droit chemin » (LESM : 85). L'âme est plus importante que le corps ! Le corps est périssable telles les fleurs de Jacques Brel dans Les Bonbons: il est voué à la désintégration totale, c'est pourquoi insister sur le travail sur l'âme est une façon de cultiver chez ces jeunes la culture de l'immortalité « afin d'endurer [leur] condition mortelle » (Ricœur, 1991: 54). Désormais, leur biographie posthume résidera dans leur œuvre ${ }^{8}$ fatale à laquelle ils ont été programmés. Le cogito cartésien, je pense donc je suis, chez lui, va totalement changer de sens, il deviendra désormais, je me tue, je tue, j'ai existé. San Juan, ou Seyf el Islam (Glaive de l'islam) de son vrai nom Khalid de Tuez-les tous et Yachine, Fouad, Nabil, Hamid des Etoiles de Sidi Moumen sont arrivés à une conclusion désolante au vu, tout d'abord, de leur condition sans issue ni portée doublée d'un ressentiment envers soi et envers la société à laquelle il n'appartiennent pas ; puis de leur endoctrinement aveugle et aveuglant: la plupart des échanges interhumains dans le système sociopolitique et géostratégique sont démystifiés, ils sont mensonge et exploitation. Cette conclusion est partagée par de grands philosophes et politiciens tels Max Weber, Hannah Arendt et Paul Ricœur entre autres. Ce dernier écrit : « Le pouvoir existe quand les hommes agissent ensemble ; il s'évanouit dès qu'ils se dispersent. La violence est l'exploitation même de cette faiblesse par un projet instrumental » (1991: 18). En écho à cette citation, San Juan dit: « Notre nation se disloque, entre en putréfaction, il faut la régénérer, avec l'aide de Dieu, ou le Diable» (TLT : 24). Dieu-Amour au coude-à-coude avec le Diable dans le raisonnement du pilote de ligne qui entame sa descente aux abysses de la haine, avec en ligne de mire, un climat d'idées des plus sombres et une existence surnuméraire que sa mort fracassante va réhabiliter.

\footnotetext{
${ }^{7}$ Mise en avant des droits humains, universalisation de la démocratie et des techniques, etc.

${ }^{8}$ Ce mot est pris dans le sens qu'a sa traduction dans l'exégèse du Coran et de la Sunna.
} 
Être, pour ces gens-là, est un idéal entre les mains des prédicateurs de la mort. Du coup, leur passage ici-bas atteindra la complétude une fois que leur existence est pensée en termes, primo d'un absolu post mortem et deuzio d'une colère qu'ils ont appris à contenir et à maîtriser pour " l'heure » fatidique. En ce sens, cette volonté de s'autodétruire pour détruire est un élan vers un absolu animé par un mouvement qui se manifeste par des impressions de haine et de désolidarisation avec le milieu, de comportements violents vis-à-vis de la famille, de paroles et de registres avec son entourage... En fin de compte, ils rompent avec tout le monde pour aller s'isoler loin du regard des ... infidèles, qui dans la montagne, qui en Espagne. L'insertion dans la société leur semble incompatible avec cet apprentissage qu'ils viennent d'acquérir et qui, désormais, va considérablement baisser le degré de leur sociabilité puisque le principe d'attache qu'ils ont avec l'État est dorénavant divin : «il avait grandi avec la volonté de la puissance et l'absence virile pour la concrétiser et il y avait ce putain d'enfant de salaud de Saoudien avec sa gueule d'apôtre efféminé, tout à la fois mère et père pour leur redonner des couilles et des poils au cul» (TLT : 82). Khalid sait pertinemment qu'il est l'instrument d'une ambition qui dépasse le cadre religieux pour aller vers le politique, mais il y va les yeux fermés. Le maître à penser est élevé à un rang qui le transcende des communs des mortels et lui confère un pouvoir quasidivin :

il disait que notre part de géhenne, nous l'avons déjà subie à Sidi Moumen et que, par conséquent, il ne pouvait rien nous arriver de pire. Mieux, la foi qu'il nous instillait jour après jour forgeait le bouclier qui allait nous permettre de franchir les sept cieux pour atteindre la lumière. Il nous décrivait chaque étape, avec ses écueils, ses tentations, ses doutes et ses errances. [...] la proximité de la lumière le plongeait désormais dans une ivresse indicible, une paix intérieure supérieure en tout point à celle que procure le haschisch (LESM, $2010: 84-85$ ).

Ainsi, boire du vin : « Une nouvelle flûte de champagne. Ça rassurait les Chrétiens, un Arabe qui boit » (TLT : 18), ou avoir une relation homosexuelle :

Le corps de Nabil frôlant le mien produisit une épouvantable érection de mon sexe. Il le prit dans sa main tout naturellement et nous nous embrassâmes. Nous nous déshabillâmes sans réfléchir et nous nous aimâmes. En silence (LESM, $2010: 127)$.

ne sont plus condamnables. L'expérience acquise par ces mentors va les aider à jouer les facilitateurs auprès des plus jeunes. Du coup, et comme par miracle, le maître va être élevé au rang d'un père idéal avec l'intention de construire un monde nouveau à leur image. Et dans ce projet d'édification de ce monde-îlot à marge, on commence forcément, et symboliquement, par une mosquée pour s'installer et se territorialiser d'une façon sacrée. On reprend les mêmes gestes fondateurs de l'Islam lors la hijra'. Tout y passe, un travail sur le culte par un apprentissage ciblé ; un autre sur le moral et le corps : "C'était une mosquée en miniature. Il y régnait ce silence propre aux

${ }^{9}$ L'émigration d'un musulman d'une terre de mécréance et /ou d'hérésie vers une terre musulmane. 
lieux du culte où la présence du Seigneur se fait ressentir un peu plus qu'ailleurs. Le salut des Samouraïs était revu et corrigé par un verset du Coran » (LESM : 104). Le sport, dans l'optique de la brigade, est un moyen pour cultiver le corps et garder la forme, tout en passant, en filigrane, les enseignements de la façon la plus ludique qui soit. Paradoxalement, ce corps-même va devenir un instrument de destruction. Cette façon de procéder a été un accélérateur de la formation idéologique qui a réussi à rallier, en faveur de sa démarche, un groupe de jeunes gens, acquis pour la cause orthodoxo-intégriste. Le sport et la religion s'associent pour socialiser des jeunes et les éduquer, et deviennent, par la même occasion, leur port d'attache. Et comme chez les sportifs professionnels, ces jeunes gens vont être sous les projecteurs, après leur auto-désintégration en exécutant l'irréparable. Le groupe a réussi à se constituer en petite communauté avec de nouvelles bases du vivre-ensemble. Opération réussie : le serment est consommé, et une confirmation de l'obligation du devoir envers le maître est en marche. Reste maintenant de respecter des diktats qui placent les prédicateurs dans une sphère qui leur confère une aura supra-humaine avec un brin de sainteté, en aucun cas, démenti. Ainsi, on démissionne d'une communauté sous le halo d'une nation pour être intégré dans une communauté sectaire très sélective sous les auspices de cet accord souterrain à facture pseudo-transcendante.

Les jeunes vont se métamorphoser en zélotes d'une conception fausse et faussée de l'islam qui est avant tout SALUT comme il a été rapporté du Coran : «"Car celui qui a tué un homme qui lui-même n'a pas tué, ou qui n'a pas commis de violences sur la terre, est considéré comme s'il avait tué tous les hommes" » (TLT: 25). Ils se laissent pousser la barbe, et de proche en proche, celle-ci devient le moyen de se rapprocher du maître et « d'entretenir les peurs et la polarisation » (Ramadan, 2009 : 154) : « Je portais la barbe pour ressembler à Zaïd » (LESM : 109) et jour après jour, la barbe devient signe visible de la barbarie : «Il nous faut nous unir et demander l'aide de Dieu. Nos barbes les font déjà trembler» (LESM : 122) et les font entrer dans un chantage imaginaire qui deviendra, avec le temps, un piège que les médias relayeront, faisant ainsi adhérer le plus de personnes possibles à l'idée que les barbus seraient un danger en puissance. L'apparence devient un signe d'appartenance comprise, de part et d'autres, en tant que message pour entretenir la différence, première étape pour le duel en vue : une pratique aussi ancienne que la politiquemême, " combien d'autres ont adopté la casquette de Staline ou la tenue de Mao » (Mimouni, 1995: 237). Commence dès lors une animosité sans précédent envers ce signe visible d'appartenance à un groupe, à une organisation, par extension. Yasmina Khadra dans Les Agneaux du Seigneur s'arrête aussi au niveau de ce "signe " ostentatoire quand son personnage Tej s'adressant à son ami Kada, il lui demande : "Quand vas-tu laisser pousser la barbe, Kada ? Le cheikh y tient. Il faut marquer la différence » (2014: 43). La même pédagogie est reprise dans ce roman. La barbe marque une certaine façon d'être dans une société qui joue, elle aussi, sur l'apparence et le paraître. Le dressage est à son paroxysme, et Abou Zoubeïr et compagnie, de proche en proche, sont des messies qui « connaissaient le coran par cœur et les paroles du Prophète comme s'ils avaient vécu dans son entourage » (LESM : 67), et dont la 
« raison d'être était de nous aider à purifier nos âmes » (LESM : 85). C'est que « La tyrannie procède par séduction, par persuasion, par flatterie » (Ricœur, 1991: 134). Des paroles du Prophète aux paroles de l'émir, que de chemin parcouru. En ce sens, il paraît clair que l'objectif ultime du prédicateur est de faire croire aux néophytes, en évoquant Dieu et son Messager, qu'Ils sont à l'origine de toute parole et responsables de ce qui va être réalisé ou programmé du fait que « La justesse d'une cause n'a pas l'immanente vertu de [...] préserver de l'injustice. Bien au contraire, la conviction $[\ldots]$ de bon droit a souvent tendance à $[\ldots]$ rendre moins vigilants » (Mimouni, 1995: 128). À cause de leur caractère subversif, elles vont permettre de brouiller le monde empirique tel que ces jeunes le comprenaient et le vivaient. Ce langage dans son usage pragmatique a impacté les jeunes pour avoir en fin de compte une dimension explosive. La méthode est simple : s'élever au rang d'éducateurs et enseigner aux disciples comment imiter sans trop poser de questions, puisque tout ce qui sera dit, ou sera fait, ou sera démontré entrent dans la case du Droit Chemin. C'est dans ce sens que la visibilité affichée est un gage d'appartenance à un clan en même temps qu'elle est un refus des lois et des textes régissant le pays, d'autant plus que cette visibilité est, en elle-même, un moyen d'exacerber les craintes de retourner l'islam contre un pays lui-même musulman. Dans ce sens, la barbe a un effet, d'une part, sur la société et, d'autre part, sur les pouvoirs publics, elle sera vue comme une menace, une atteinte aux règles de droit et un moyen sectaire pour vivre la religion.

Le charisme de l'émir et des représentants de l'Organisation est tel qu'ils deviennent des Idoles dans l'absence de modèle à suivre, situation de détresse que les mentors vont exploiter : " il restait mon idole au même titre que Yachine, mon maitre à jouer » (LESM : 88). La mise en œuvre de la cellule d'embrigadement a eu ses fruits et le résultat est palpable. Les jeunes oubliés de Sidi Moumen sont hypnotisés et manifestent, désormais, un état de sujétion avancé sur tous les plans psychique et moral les privant d'une partie de leur libre arbitre, de leur volonté conduite, a priori, par la raison, mais qui s'est anéantie à force d'abrutissement méthodique : " Nous étions ses soldats. L'heure du jihad était arrivée. Il nous félicita d'avoir été élus par le Seigneur pour mener à bien ses volontés » (LESM : 140). Les banlieusards troquent leur désœuvrement contre le ralliement à la cause des barbus dont le discours rôdé a eu raison de leur raison et de leur discernement : il va modifier en profondeur les comportements des jeunes au niveau de la pensée et du discours au point de ne plus accepter que l'Autre soit en contradiction avec leurs principes. En fait, un travail de lavage de cerveau a été initié et a abouti à leur abrutissement, et si Hegel devait s'immiscer dans cette affaire, il conclura que ces jeunes sans avenir n'ont plus de Raison puisqu'ils refusent, de facto, la contradiction.

Cette attitude négationniste de son propre chef se manifeste dans les faits par l'adoption d'une nouvelle ligne de conduite, en même temps que le rétablissement méthodique et rigoureux d'une identité au diapason de celle du passé. Puisque l'enseignement progressif de ces jeunes, jusque-là, ignorants et fragiles, repose, avant tout, sur le consentement irréductible aux dires et aux agirs des émirs qui sèment, désormais, le vent et le froid au sein du groupe, on ne peut qu'être sceptique par 
rapport à leur évolution. Ils vont apprendre à survoler négligemment leur passé, comme s'ils n'y trouvaient que la médiocrité et la mesquinerie. L'émir Zïad, Abou Zoubeïr, les frères Oubaïda et les autres représentants de toutes les organisations terroristes vont réussir un tour de passe-passe en faisant croire à leurs disciples qu'ils étaient en train de les corriger et de les remettre en état... en état de détruire tout ce qui ne leur ressemble pas : « (les tours jumelles figuraient dans son esprit les deux arbres défendus) » (TLT : 25) qu'il fallait approcher brutalement comme l'avaient déjà fait Adam et Ėve.

Une nouvelle ère doit naître. Et c'est de l'Apocalypse que naîtra un nouvel ordre mondial : «Demain, ils jouiraient de l'Apocalypse. Belle, esthétique, tout le monde la contemplerait; une œuvre d'art, passée en boucle sur toutes les télévisions du monde » (TLT : 20), « Ainsi débuta notre sombre glissade dans un monde qui n'était pas le nôtre. Un monde nouveau où nous allions peu à peu nous enliser et qui finirait par nous engloutir une fois pour toutes » (LESM : 102). On passe, et ce sans transition, d'un monde régi par une des formes de pouvoir dans des configurations diverses à un monde régi par des manifestations sporadiques de la puissance légitimée par des personnes qui se disent proches de la Parole divine instituant ad hoc une acceptation totale et sans tergiversation ni réflexion!

Le profil psychologique de San Juan qui lui arrive même de rire de ce surnom « accolé à l'Apocalypse » (TLT : 79) et les autres kamikazes de Sidi Moumen est problématique. Il n'est guère « orthodoxe », il est plutôt « radical »: "S'il n'avait pas été aussi radical, l'Organisation ne l'aurait jamais compté parmi les siens. Ils sentaient bien qu'il n'était pas un croyant orthodoxe. Mais, ils aimaient ça. Ils répétaient souvent que les plus sceptiques étaient sur la voie de la vérité » (TLT : 13). Le radicalisme est une condition sine qua non pour atteindre le statut de martyre. L'Organisation ${ }^{10}$ va " pratiqu[er] un terrorisme radical [et...] provoquer[a] partout une peur panique» (Mimouni, 1995 : 250). Et en sa qualité de futur martyre, il peut profiter hic et nunc de toutes les jouissances offertes «ils lui avaient répété qu'à présent, quoi qu'ils fassent, ils seraient accueillis au paradis, ils entoureraient tous les prophètes et boiraient tout leur saoul en baisant des vierges ; alors autant prendre de l'avance : régime d'exception pour les martyrs » (TLT : 17). C'est ce « même régime d'exception » qui va autoriser Seyf El Islam à errer dans les rues dans les nuits blanches de Portland avec une prostituée qu'il ne va pas toucher. Ses relations avec les femmes sont entachées avec des souvenirs de son enfance et de sa mère. La déchéance morale et religieuse dans laquelle il se trouve est à l'image de sa destruction et son atomisation, il a depuis cette décision mortifère un «esprit incandescent $[\ldots]$ au souffle divin et démoniaque » (TLT : 92).

Sans s'en rendre compte, et en... bons disciples, ils vont lancer une des critiques les plus virulentes à l'égard de la mondialisation et de sa société fondée sur une éthique qui ne ressemble en rien à ce qu'ils croient être une société musulmane.

${ }^{10}$ AlQaïda 
Comme conséquence logique à ce système de pensée qu'ils adoptent et qu'il croient être émanant de l'ordre divin, ils vont se dire qu'ils doivent rendre justice par euxmêmes, condamner et jouir de cette souffrance qu'ils vont créer avant même l'exaction de l'acte fatal, libérateur et purificateur à la fois : " Je te vengerai de ceux qui ont pillé ton enfance et englué tes rêves dans la boue. Je leur ferai payer rubis sur l'ongle les années d'asservissement qu'ils nous ont fait subir...» (LESM : 143). Ils ont produit un ennemi qui va cristalliser toute leur énergie maléfique et meurtrière.

La souffrance, telle vécue par les kamikazes, est passée par plusieurs étapes jusqu'à germer, en fin de compte, pour donner à voir l'horreur puis la pourvoir. Elle est d'abord psychique (égologique), physique (géolocalisable, du moins pour le héros des Etoiles de Sidi Moumen), puis liée au non-désir, phase de désespoir fatal à tout épanchement vers une vie humaine normale au sein d'une famille. Naîtra de ce dernier point une souffrance relationnelle des plus destructrices, pour aboutir, en dernier ressort, à cette souffrance donnée et reçue à la fois en spectacle en temps réel : « le temps réel des images » (Baudrillard, 2002:25) : «Il ne lui restait qu'une heure à vivre, une heure pour dresser les hommes les uns contre les autres [...] et affirmer sa toute-puissance pendant que l'avion descendait, descendait avec lenteur puisqu'il vivait le présent figé de la damnation, comme en un rêve » (TLT : 121). Le pilote se prépare indubitablement au tragique de sa situation et de celle de l'humanité depuis sa radicalisation. De l'autre côté de l'Atlantique, au Maroc, les Jeunes kamikazes « à la foi tardive » (Mimouni, 1995: 280) vont connaitre « une jeune mort, aussi. Une mort presque sans cadavre, car le mien a été ramassé à la cuillère. L'ironie, ils ont enterré avec moi des restes de Khalil : une mâchoire édentée, deux doigts de la main droite, celle qui avait actionné le dispositif » (LESM : 61).

En effet, l'accès à la connaissance et à la conscience est ce cheminement-même qui va plonger les bombes humaines dans l'abîme et l'obscurité des idées noires qui rendent la vie désir de mort et de vendetta remontant aux Croisades. Ils connaîtront une vie posthume des plus médiatisées et occuperont, en conséquence, une dimension planétaire riche d'antipathie et de haine mortifère, à l'égard d'eux-mêmes et à l'égard de ce qu'ils représentent sur le plan religieux. Avec cet acte ultime, ils esquissent une méta-vie à mi-chemin entre néant et être, au service d'une cause au-delà de l'entendement des communs des mortels. Camus aurait vu que ces suicidaires ont accompli « une grande œuvre » (1984:16) qu'ils ne verront guère. D'ailleurs, Seyf El Islam, ou Khalid ou San Juan «était heureux [...] et fier de détenir, parmi les derniers sur la terre, la puissance orgiaque de la Parole » (TLT : 92) où ils pouvaient faire danser Dieu et Satan sur un requiem des plus macabres. Ce processus fait le deuil d'un avant et installe le concerné dans un traumatisme cumulatif célébrant la mort sui-donnée et la gloire posthume. Cette pulsion silencieuse de la mort est une réponse fracassante, en amont, au vide psychique et sociétal dans lequel vivent les protagonistes. Elle est cette «surdité de l'économique libidinale aux règles de la composition, à la hiérarchie de l'organisme» (Lyotard, 1994 : 187). En tant que citoyens, ils ne peuvent que tourner le dos au rythme de la vie moderne. Leur adaptation est impossible, et leurs habitus, en conséquence, seront revus et corrigés 
en fonction de ce nouvel apprentissage de la vie qu'ils reçoivent d'entre les mains de prédicateurs lisant à leur guise le Coran. Dorénavant, ils sont absorbés par des idéaux extrêmes; et on va leur construire un monde à leur image tout en les poussant à se doter d'une attitude d'esprit suicidaire envers l'Autre.

D'ailleurs, cette lecture ne prend pas en considération l'évolution de la société et du monde qui nous entoure. Tout se déroule au détriment d'une vision avec un intérêt critique prenant en compte ces paramètres de relations humaines mouvantes dans toutes ses configurations : économiques, politiques, culturelles, religieuses... Une avalanche de faits inhabituels et disparates vont agir sur l'appréhension du monde qu'ils ne seront plus à même de rester eux-mêmes. Les jeunes, sans instruction et sans avenir, seront une proie facile à racoler. Ils subissent à leur insu une manipulation mentale qui les mènera simultanément à la mort et au meurtre dans un acte démesuré et disproportionné, avec une visée symbolique qui veut semer la terreur et le doute chez la population, d'un côté, et chez les pouvoirs publics, d'un autre côté : «New York était la ville des iniquités, le world Trade Center le symbole de l'orgueil démesuré de l'Amérique, et du saccage du monde les tours, les démons Gog et Magog qu'il fallait abattre... » (TLT : 89).

L'Histoire va alimenter un ressentiment enfoui qui n'attendait que le jour de se manifester post-mortem avec, en clef, une haine indifférenciée à toute personne qui ne partagerait pas les mêmes principes, et même ceux qui appartiennent à leur même communauté. Tout le monde est coupable de ne pas s'être rangée du bon côté, c'est pourquoi il faut les tuer tous sans aucun remords, parce que selon eux, la Sourate II, V 154 parle d'eux et en est la preuve ultime : " "Ne dites pas de ceux qui sont tués dans le chemin de Dieu : 'Ils sont morts !' Non !... Ils sont vivants, mais vous n'en avez pas conscience"» (TLT : 22-23).

\section{Du rapport avec l'État}

Se pose, dans ces conditions de perte de substance, la question de l'orientation de la fidélité de l'individu : serait-elle envers l'État ou envers Dieu, sachant que dans bien de pays, le droit civil et pénal réunis, se basent sur des commandements divins. Dans ce contexte, l'intéressé est un être de l'intérieur, introverti qui se définit par rapport à sa vérité profonde selon des représentations psychotiques avec des scénarii intérieurs, déterminant une essence au diapason de la société à laquelle il devrait juridiquement appartenir. Schématiquement, ces orthodoxes répondent en écho à Mauss quand il décrit le schisme à l'intérieur d'un territoire qu'on croit une nation : «Une société, c'est un individu, les autres sociétés sont d'autres individus. Entre elles il n'est pas possible - tant qu'elles restent individualisées - de constituer une individualité supérieure » (Mauss, 1969 : 34). Le tragique, à deux niveaux superposés, s'installe, celui à l'échelle de l'individu et celui à l'échelle plus grande : la société. Cet état va faire naître un vide allant jusqu'à la perte de soi et la volonté toujours grandissante que les répercussions de ce nihilisme atteigne la société impie ! 
L'espace intime de réflexion va situer le pensé et le vécu comme une obsession. En conséquence, l'acte final résultant de ces remue-méninges sera, après coup, une preuve d'objectivation d'une idée qui a germé le long d'un processus de rhinocérite. Il devient un être ne manifestant pas de désir, il est là à subir et à acquiescer les avis et les opinions des autres sans réserve aucune, s'opposant ainsi à son statut d'Homme dont « La représentation d'une fin désirable suscite la délibération. [...] c'est-à-dire examiner les raisons pour et contre l'action projetée. Normalement, la délibération se termine par un jugement que nous pouvons schématiser ainsi : il est raisonnable - ou il n'est pas raisonnable - d'adopter le projet soumis à l'examen » (Foulquié, 1972 : 32) Il n'est plus libre, et cette absence nuit essentiellement à sa capacité d'entendement qui l'aveugle et la handicape face à son réel et à sa condition... d'homme.

Le rien va s'installer au sein même de ce qui constituait foncièrement le vivre en commun. En ce sens, le commun devient par miracle et par jeu phonétique commeun, qui vite se métamorphosera en un. En conséquence, le pouvoir s'installe entre les mains non de la collectivité ou de la communauté, mais entre les mains d'une personne libre, libre jusqu'à l'anarchie, « Cette condition fondamentale de l'homme face à ses possibles, c'est précisément sa liberté, liberté vertigineuse à laquelle chacun dans sa finitude et sa singularité est voué »(Verrecchia, 2004:143).

Les deux romans y répondent en affirmant que le serment de " ces gens-là » est formellement en direction d'un ordre divin, celui-là même qui a institué le " "protocole de tolérance" à une époque où les sociétés chrétiennes ne toléraient rien. Pendant des siècles, ce "protocole" fut, dans le monde entier, la forme la plus avancée de coexistence » (Maalouf, 1999: 79). En effet, c'est pendant cette période que Charles IX recommandait à ses soldats de tuer des protestants venus célébrer le mariage de Henri de Navarre : « Tuez-les, mais tuez-les tous pour qu'il n'en reste pas un pour me le reprocher » c'est à vrai dire un massacre, et c'est au nom de la religion qu'on a procédé ainsi. Bachi va, entre autres, exploiter cette sentence meurtrière pour forger son titre. Mais celui-ci a d'autres occurrences dans la littérature, dans la musique et dans le cinéma ${ }^{11}$.

\footnotetext{
${ }^{11}$ L'intitulé du roman Tuez-les tous ! fait référence au premier album du groupe de musique Metallica, intitulé «Kill'em All» diffusé en 1983. Dans la culture cinématographique, le titre trouve un autre écho dans un western spaghetti de production espagnole et italienne de 1968 intitulé AMMAZZALI TUTTI E TORNA SOLO (Italie), Tuez-les tous et revenez seul. Au cinéma, la dernière occurrence est dans le film Le Royaume, film américano-allemand réalisé par Peter Berg, sorti en 2007. La phrase est répétée deux fois par deux acteurs différents représentant chacun deux idéologies différentes.
} 


\section{Médias et Histoire}

Dans les faits, à partir de la deuxième partie du $\mathrm{XX}^{\mathrm{e}}$ siècle, le monde arabe commence à changer à une vitesse au rythme du pétrole et de la dictature qui est allée avec. La vie quotidienne, elle-même, va se trouver au centre d'une métamorphose où tout devient facile à avoir tant que l'argent coule de source. Puis, comme si c'était dans l'ordre des choses, la société arabo-musulmane va produire une religion à son image : « car il faut bien se souvenir que les sociétés, comme toutes choses naturelles, ne changent réellement que si leur milieu change, et n'ont en elles-mêmes que des forces relatives d'altération » (Foulquié, 1972:35). Et si « La "société occidentale a inventé l'Église et la religion dont elle avait besoin » (Maalouf, 1999: 85), le monde musulman va faire pareil, mais dans l'autre sens comme si l'on était devant la valeur inversée des Valeurs. Dorénavant, le monde occidental va virer vers une pseudotolérance d'apparat; et le monde arabe vers... ce que les médias appelleront le terrorisme, qui est, il faut le dire, une création occidentale. Bref, il y a naissance, désormais, d'une contre-puissance en réaction à l'occident. Bachelard aurait pu répondre à cette conclusion médiatique en disant :

Il y a des idées si rapides qu'elles ne sont plus des idées actives. Telle est l'idée de néant. [...] Ainsi Néant, Vide, Rien, Non se manient comme papier déchiré. La négation est tout de suite opérante. Elle permet au penseur une volte-face dans le règne de ses idées. Elle le fait autre à bon marché, sans peine, sans responsabilité, en un trait de plume (Bachelard, $1988: 11$ ).

Dans cette campagne anti-islam, l'Occident va profiter de sa suprématie technologique et médiatique pour diffuser sa haine contre l'islam en essayant de prétendre qu'il n'est pas contre les musulmans! A vrai dire, l'Occident va trouver dans les médias actuels le deuxième homme dans le même sens donné à quelqu'un comme Saint Paul chez les chrétiens ou Omar chez les musulmans. La diffusion du fait religieux sera assurée par cet homme; en parallèle, les médias, surtout la télévision puis Internet, seront le deuxième homme au service de l'Idéal Occidental. La vampirisation de l'Islam, pris en tant que cheval de bataille de l'Occident, sera mal vécue par les musulmans au point qu'ils vont réexploiter les mêmes médias, dans l'objectif d'une réaffirmation de l'Islam soit sur leur territoire (le Maroc): « Il [l'émir] dit qu'il n'y avait pas lieu de craindre les ennemis de l'islam, nous tenions nos destins et les leurs au bout d'un fil. Il suffisait de le tirer pour les expédier en enfer » (LESM : 140) ; soit ailleurs, outre Atlantique (États Unis), tout en installant cette action morbide dans le sens d'une vengeance sur deux siècles de soumission et de suprématie occidentale : «Et c'était bien ce qu'il allait faire demain, se jeter dans le brasier pour que le monde entier, horrifié par son acte, contemple son incrédulité. Il deviendrait ainsi l'instrument damné de Dieu. Il briserait l'orgueil de l'Amérique pour quelques siècles » (TLT : 27) en rompant à jamais ce que Bourdieu appelle les « capitaux sociaux » (Bourdieu : 1980). 


\section{En guise de conclusion}

Les deux romans racontent comment de jeunes gens vont se faire exploser pour une cause à laquelle ils ne comprennent absolument rien. Ils ont été apprivoisés par des maîtres à penser avec des allures d'anges. En fin de compte, ils vont précipiter leurs disciples vers une mort sans cercueil et sans cadavre. Pour arriver à leur fin, les prêcheurs sont arrivés à construire des machines téléguidées au service d'une idéologie rétrograde, qui pointe vers la tache aveugle, et dont le seul but est d'éliminer tous ceux qui ne leur ressemblent pas, puisque, de facto, ils sont contre eux et « qu'il fallait des hommes de [leur] espèce et que Dieu comprendrait» (TLT : 58). Désormais, la société à laquelle ils aspirent est celle de l'identique, de l'absolu-même. La différence n'a plus lieu de cité, un nouvel univers est en cours d'édification dont les termes se résument en une formule injonctive « Tuez-les tous! ». Tous les Autres sont passibles de cette sentence aussi ancienne que l'Homme: elle constitue la représentation d'un modèle irrationnel qui fait la belle part à l'unidimensionnel.

L'horrification s'intensifiera avec l'immixtion attendue des médias qui vont rendre encore plus sensationnel le sensationnel lui-même. L'acte perpétré est hors de commun et révèle le désarroi de ces kamikazes, qui au lieu de consacrer leur vie à semer la vie, ils vont, au contraire, semer la mort avec une volonté inconsciente à se débarrasser de leur humanité dans un élan «qui témoigne de l'effroi d'observateurs emportés par la puissance de leurs affections et incapables de connaitre un phénomène qu'ils cherchent à décrire » (Le Cour Grandmaison, 2002 : 65).

\section{RÉFÉRENCES BIBLIOGRAPHIQUES}

Bachelard, G., (2010) Fragments d'une poétique du feu. Paris, PUF.

Baetens, J., (2005) Romans à contraintes. Amsterdam-New York, Rodopi.

Baudrillard, J., (2002) L'Esprit du terrorisme. Paris, Ed Galilée.

Bourdieu, P., (1980) «Le capital social », in Actes de la recherche en sciences sociales. $\mathrm{N}^{\circ} 31$, pp. 2-3.

Camus, A., (1981) Le Mythe de Sisyphe. Paris, Gallimard, Coll. Idées.

Foulquié, P., (1972) La Volonté. Paris, PUF, Coll. Que sais-je?

Khadra, Y., (2014) Les Agneaux du Seigneur. Paris, Pocket.

Le Cour Grandmaison, O., (2002) Haine(s), philosophie et politique. Paris, PUF.

Lyotard, J-F., (1994) Les Dispositifs pulsionnels. Paris, Galilée « coll. » Débats.

Maffesoli, M., (2001) « La violence et sa ritualisation », in Watthée-Delmotte, M. (dir.), La Violence et ses ritualisations. Paris, L'Harmattan.

Maalouf, A., (1999) Les Identités meurtrières. Paris, Grasset.

Mauss, M., (1969) La Nation. Paris, Les Éditions de Minuit, Coll. Le sens commun.

Mimouni, R., (1995) La Malédiction. Tunis, Eddif-Cérès.

Onfray, M., (2001) Antimanuel de philosophie. Paris, Bréal- Rosny.

Ramadan, T., (2009) Mon intime conviction. Paris, Archipoche. 
Ricœur, P, (1991) Lectures 1, Autour du politique. Paris, Seuil, Coll. La couleur des idées.

Seymour, M., (1999) La Nation en question. Montréal, L'Hexagone.

Verlaine, P., (1991) Fêtes galantes et Romances sans paroles, précédé de Poèmes saturniens, Paris, nrf, collection Poésie/Gallimard, Préface et notes de Jacques Borel.

Verrecchia, B., (2004) "Le Devant-quoi de l'angoisse», in Chamond, J., Les Directions de sens, Phénoménologie et psychopathologie de l'espace vécu. Paris, Vrin, Coll. Phéno.

Le Petit Robert de la langue française 2012, Edition électronique.

\section{CORPUS}

Bachi, S., (2006) Tuez-les tous. Paris, Gallimard.

Binebine, M., (2010) Les Etoiles de Sidi Moumen. Casablanca, Le fennec. 